\title{
Development of a hybrid AHP and Dempster-Shafer theory of evidence for project risk assess- ment problem
}

\author{
Saad M. Albogami ${ }^{a}$, Mohd Khairol Anuar Bin Mohd Ariffin ${ }^{a}$, Eris Elianddy Bin Supeni ${ }^{a}$ and \\ Kamarul Arifin Ahmad ${ }^{a}$
}

${ }^{a}$ Universiti Putra Malaysia, Malaysia

\begin{tabular}{l}
\hline C H R O N I C L E \\
\hline Article history: \\
Received: February 1, 2021 \\
Received in revised format: Octo- \\
ber 2, 2021 \\
Accepted: October 29, 2021 \\
Available online: \\
October 29, 2021 \\
\hline Keywords: \\
Project Management \\
Project Selection \\
Dempster-Shafer Theory of Evi- \\
dence \\
AHP \\
Uncertainty
\end{tabular}

\begin{abstract}
A B S T R A C T
In this paper, a new hybrid AHP and Dempster-Shafer Theory of Evidence is presented for solving the problem of choosing the best project among a list of available alternatives while uncertain risk factors are taken into account. The aim is to minimize overall risks. For this purpose, four groups of risk factors, including Properties, Operational and Technological, Financial, Strategic risk factors, are considered. Then using an $\mathrm{L}_{2}{ }^{4}$ Taguchi method, several experiments with various dimensions have been designed and solved by the proposed algorithm. The outcomes are then analyzed using the Validating Index (VI), Reduced Risk Indicator (R.R.I\%), and Solving time. The findings indicated that, compared to the classic AHP, the results of the proposed hybrid method were different in most cases due to uncertainty of risk factors. It was observed that the method could be safely used for selecting project problems in real industries.
\end{abstract}

\section{Introduction}

The manufacturing industry plays a crucial role in each country's economy (Delgoshaei et al., 2017). Developed countries mostly have better industries and, therefore, investments in potential opportunities in the industries (manufacturing projects) accordingly. For example, Абдикеев et al. (2019) reported that in 2017, 30\%, 28\%, 20\% and 13.5\% of the annual GDP of China, South Korea, Germany and Russia belong to the manufacturing industry. Of this, a significant value belongs to manufacturing industries (Dašić et al., 2019). In today's rivalry, choosing appropriate decisions plays a key role in a manufacturing firm's success. In most cases, choosing an inappropriate choice will impose detrimental effects on a company or cause project failure (see section 1.3). Risks are considered an inseparable part of a project and thus should not be ignored. Each year, many projects have failed due to the harm that they impose on them. Risks attributed to projects can have various sources but all have the same goal: to fail a project. Fig. 1 depicts the correlations between the level of risks associated with a project and the amount at stake throughout the lifecycle of a project. As shown by Fig., the level of the risks at the earlier phase of a project is significantly higher than what in the ending phases. Such a fact can reveal the importance of risk management in a project selection. Carvalho \& Rabechini Junior (2015) also mentioned a significant correlation between the level of risk management taken by a project team and project success.

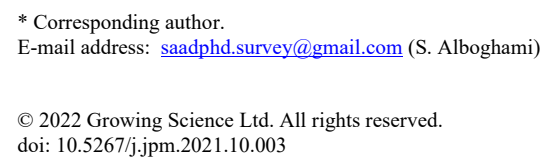




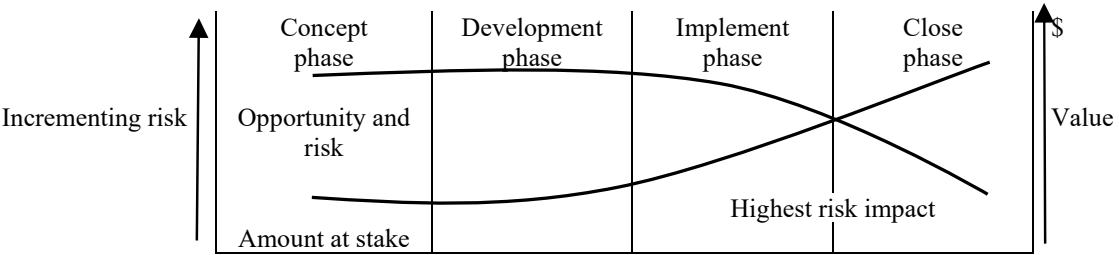

Fig. 1. Correlations Between the Level of Risks Associated with A Project and the Amount at Stake

In the project management body of knowledge (PMBOK), which is considered the essential guideline in the project management field, risk management is considered one of the $9^{\text {th }}$ areas of project management. Therefore, minimizing the risks of a project is a vital need. Subsequently, the more attention pays to risk identification; the less risk will be faced during the lifecycle of a project. For this purpose, and as will be seen in the literature review, efforts have been made during the last two decades to propose various decision-making methods to investigate different risk management problems. Of these, a noticeable share belongs to the project selection problem. To continue, the shortcoming of the current research methods will be investigated. However, in the literature review, the problem will be explained in more detail. The main question here is whether deterministic decision-making methods can satisfy all Risk Management needs in project Selecting problems. In continue, this question will be answered. Considering the uncertainty in the decision science discipline challenges the use of classic methods. For example, the classic Analytic Hierarchy Process (AHP) considers constant values for alternatives and factors and chooses the best alternative accordingly. While considering the term uncertainty, each cell in the AHP will not have a specific value necessarily, and instead, a range of values (confidence intervals) must be estimated by considering confidence levels $(1-\alpha)$. Therefore, an optimum solution will not necessarily exist and can be changed by considering different confidence intervals. Using the facts mentioned above, there is ample evidence available to consider uncertainty in selecting the best project among the available alternatives to minimize the project's risks in the executing phase. Therefore, in this research, a new decision-making model will be proposed to select the best alternative while uncertainties exist for the level of risk factors. The aim will be to minimize the risks associated with the company's capabilities such as available money, human resource skills, machinery, documentation systems and quality control level. This study can help industrial business owners to select appropriate projects according to their capabilities and strengths. Choosing the wrong project for construction can have detrimental effects on a business and impose irreparable financial harm. In each county, there can be found many failed projects that remain unconstructed for years or even decades. The same phenomenon happens in the industry. A quick search in each industrial zone, a series of unsuccessful projects, is left alone simply because the managers believe that the projects will not be successful and make profit even if they are successfully working in the future. They may also simply because they made a wrong decision on choosing projects and calculating the required budget for completing them due to not paying enough attention to selecting appropriate projects according to the money and resource availability.

In this research, a new method will be proposed to involve uncertainty of available evidence in the process of selecting the best alternative among a list of available proposals of manufacturing projects in order to minimize the level of the risk factors associated with a project by utilizing scheduling and line balancing risk assessment.

\section{Literature review}

In Multi-Attribute Decision Making Methods in Project Scheduling (MADM) methods, more than one attribute is usually considered. The most preeminent advantage of MADMs (comparing to MODMs) is that they are easier to be understood by managers in real practice. Moreover, their outcomes are more tangible and can easily be applied directly in real manufacturing systems. Methods like the Analytical Hierarchy Process (AHP) and The Technique for Order of Preference by Similarity to Ideal Solution (TOPSIS) are among the most critical MADMs. MADM methods can usually be hybridized with other methods to enhance their functionality. In this section, several MADM references are investigated, and essential questions regarding MADM in risk management will be answered. MADM methods are regularly used in project management problems. Kuo and Lu (2013) proposed a fuzzy MCDM method for assessing risk factors associated with metropolitan construction projects. The proposed method used a multiple fuzzy attributes direct rating scheme to measure the occurrence probability of risk factors. Marcelino-Sádaba et al. (2014) proposed a risk management method for project management in small manufacturing systems. The method is then applied to 72 companies located in Spain. Their method was based on checklists and other simple tools to measure indicators and outline the corrective actions. Leu and Chang (2013) argued that although many papers used classic methods while safety factors come into consideration, most of such methods could ineffectively address the correlation between dependencies of safety factors and occupational accidents. Hence, they proposed a new safety risk-assessment model based on Bayesian networks and fault tree concepts. Their outcomes show that the proposed method can effectively address the safety management of a project. Hossen et al. (2015) used a construction schedule delay risk assessment method for nuclear power plants where a hybrid AHP and Relative Importance Index and schedule delay risk are considered. Kim (2015) developed a new model for risk management which is designed based on Bayesian rules. In their model, the pre-project cost risk assessment and actual performance data were used to portray a range of possible project costs at a pre-defined confidence level. Esmaeili et al. (2015a) presented an attribute-based risk identifi- 
cation and analysis method to improve available construction safety management methods, which help schedulers and designers to realize, identify and then model safety risk independently of specific activities or building components. Their method showed high performance while it was used to identify and measure a number of injuries and fatalities in construction resulting from a finite number of hazardous attributes of the work environment. In the same year, Esmaeili et al. (2015b) claimed that identifying and measuring risks associated with virtual construction work environments (which usually cause work injuries) is vital for pre-construction safety management. They proposed a 2-stage model that worked based on a principal component analysis of safety attributes and leading principal components that used potential predictors in a generalized linear model. The outcomes of applying their model indicated that by giving identifiable characteristics of planned work, it can successfully forecast the probability of a safety incident.

Islam et al. (2017) focused on using an analytical network process (ANP) on project risk management and argued that fuzzy ANP has limited functionality while incorporating new information into the risk structure. Therefore, they proposed a new fuzzy Bayesian belief network (FBBN) and showed its superiority to supersede the existing fuzzy ANPs. Valipour et al. (2017) focused on occupational accidents reported in metropolitan excavations in significant cities. To investigate this case, they proposed new criteria for risk assessment by using adopted SWARA (Step-wise Weight Assessment Ratio Analysis) and COPRAS (Complex Proportional Assessment) methods. Then, using a field study, they found that construction safety, unfavorable geological conditions, shortage of managerial experience, preliminary emergency plan and subsidence of ground are the most significant risks in excavation projects. Williams (2017) declared that systematicity causes difficulties in evaluating risk levels of complex projects. They also mentioned that one important activity to do after identifying risk is to pursue its causal chain. They outlined the steps of analyzing the systemic nature of risk and how owners and constructors can fully understand the consequences of their actions.

\section{- Do the relations between human factors influence the risk levels in networks?}

Fabricius and Büttgen (2015) argued that the manager's overconfidence directed them to the project fails in many cases. Therefore, they used risk assessment to measure overall anticipated project success and how overconfidence will influence such assessments. Using data from 204 project managers, they outlined a standardized, case-based survey and proved that overconfidence reduces risk awareness among project managers, leading them to assess risks more optimistically and more positive conclusions about anticipated project success. Wang et al. (2016) addressed a new method for construction project managers to perceive risk. They investigated the project managers' behavioral factors such as extraversion, agreeableness, and conscientiousness influence risk propensity and their implications to see if they differ in ways of perceiving risk. They found that extraversion, agreeableness, and conscientiousness impose detrimental effects on risk perception. Chemweno et al. (2015) focused on implementing appropriate risk management on the health level of assets. They showed that choosing the proper risk management approach will positively affect maintenance decision-making by identifying, analyzing, evaluating, and mitigating equipment failures. For this purpose, they offered a new risk assessment using generic selection criteria for the FMEA, FTA, and B.N. In their method, the available criteria were prioritized using ANP. Kokangül et al. (2017) dealt with solving the health and safety problem in workspaces. For this purpose, they proposed a new risk assessment method which lies on the Fine Kinney method and AHP for a large-scale manufacturing company. Then the correlation between Fine Kinney risk assessment method and AHP has been examined. Identifying risks and evaluating them is a vital step in the early stage of a project. Yet et al. (2016) addressed a hybrid dynamic Bayesian network modeling framework for analyzing risk scenarios and budget policies in agriculture projects where both uncertainty and variability of risk and economic factors were taken into account. Yang et al. (2019) argued that many risks associated with R\&D projects make them too sophisticated while standard methods are used to examine the performance of a project. Therefore, they proposed a predictive evaluation framework where belief rule-based with random subspaces were used in order to assess risks in R\&D projects. They applied their model in a number of projects in China and showed that while using their method; prominent results with prediction accuracy were achieved. While considering more than one objective function is important, Multi Objectives Decision making Models in Project Management (MODM) methods are promising ways to overcome the difficulties of super-complex project management problems. Many project management problems are Np-hard by their nature which means normal optimizers like LINGO and GAMS cannot quickly solve them. The complexity of such problems will be increased while more than one objective function is taken into consideration. This fact matches with real circumstances of projects. In real projects, managers normally consider more than one objective at the same time while selecting a project. For example, they may want to find out which project will have more profit, have fewer costs and impose fewer risks during the execution phase. Some of the most important references that used MODM techniques in the Project solving problem will be reviewed. Ansarifar et al. (2018) focused on rapid responding (time) and cost of services objectives to find the optimum location for ambulance stations and helicopter ambulances. For this purpose, they proposed a new heuristic method to solve the developed multi-objective model.

\subsection{Risk Management and Evidence Theory in Project Management}

\subsubsection{Risk Management and Its Importance in Project Management}

Cambridge dictionary defined the term risk as "the possibility of something bad happening" . This means that projects (similar to other industrial sectors) can suffer from the risks if risk factors are neglected.

\footnotetext{
${ }^{1}$ https://dictionary.cambridge.org/dictionary/english/risk. Retrieved in 30.10.2020.
} 
Risk Management is an important part of the Project Management Body of Knowledge (PMBOK) which is the most essential reference in project management worldwide. In this reference, risk assessment is divided into five main parts: Risk Identification; Risk Evaluation; Risk Analysis; Risk Planning, Risk Response. Zhang and Fan (2014) proposed a model to integrate project cost, schedule, and quality to choose the risk response strategy selection problem. They declared that by finding the optimum solution, the most appropriate risk response strategies could be taken.

\subsubsection{How to outline risks associated with a project?}

One important reason to face risks that usually cause project failures is ignoring paying enough attention to potential project risks during anticipating project elements (Fabricius \& Büttgen, 2015). It is worthy of knowing that risk identification has a significant impact on a company's future strategies. Abd El-Karim et al. (2017) focused on the added value of risk assessment, risk strategy and plan analysis to the construction industry in Egypt. They aimed to identify and measure the effect of the factors that have negative influences on time and cost contingency. Liu et al. (2017) argued that incorrect investment decisions are the main root of many losses to a project's investors. Although using quantitative risk assessment, which project owners frequently apply, can ameliorate such problems the classic risk assessment methods usually ignore assessing the effects of risk events, such as product sales falling short of expectations. Therefore, they proposed a modified version of the quantitative risk assessment model, enabling managers to outline the direct correlations between risk events and other decision variables in investing in a project. In this research we will use the risks that are classified by DELGOSHAEI (2016). However, it is essential to know that the risk identification level depends on the project's nature. While for some projects, it is essential to use complex techniques to understand the risks and the correlations between them, for others, more straightforward methods are more useful. For example, Bowers and Khorakian (2014) stated that innovative projects are riskier to be successful due to their nature. Current risk management methods might have too stern a look for innovative projects, which may damage the creativity in an innovative project accordingly. To overcome this barrier, a new framework was offered to use the generic innovation process in the risk management process of a project to outline a stage-gate innovation process model to provide an effective interface for incorporating project risk concepts.

- Does the size of a company have an impact on choosing a risk management approach?

A critical point about risk management is the contribution of the size of a company with risks and risk assessment methods. Brustbauer (2016) investigated risk management in small and medium-sized enterprises using a field study. Their outcomes indicated that using an active or a passive risk management approach has influenced choosing of offensive or defensive strategy for the studied cases, respectively. Besides, while the firm's size came into consideration, the sector's affiliation and ownership structure would also influence the implementation of risk management.

\section{- What is the role of the activities' complexity in a company in risk identification?}

Fang et al. (2016) stated that complexity usually causes barriers in identifying and assessing risks associated with a project. To overcome such difficulty, they used an important measure technique in project risk management. The complex project risk network modeling and providing complementary analysis results are used to measure risks' interactions. Tao et al. (2018) stated that location and congestion of activities must be considered during project schedule.

\subsubsection{Risk assessment methods in Project Management}

An in-depth review of the opted research studies (as we will see in continuation) showed that the most essential methods are mathematical modeling, MADM, MODM, field study and statistical analysis, and heuristic and meta-heuristics also reviewing case studies (regardless of their priority). In continuation, several important research studies are shown where risk management is the main aim. Gutjahr (2015) used a branch and bound searching algorithm for a multi-objective scheduling method while minimizing project time, and costs are considered the model's main objectives. Wu et al. (2014) provided an in-depth review of tools and methods used by researchers for business intelligence risk management. Risk can be defined as a measurable part of uncertainty (Dziadosz \& Rejment, 2015) by considering the occurrence and severity of the damage. However, uncertainty is defined as "a situation, in which something is not known, or something that is not known or certain"2. Uncertainty can increase the harms of risk or increase its occurrence likelihood.

\subsubsection{Uncertainty and Evidence Theory in Project Management}

While risk management (including risk identification and risk assessments) comes into mind, one major shortcoming is that researchers considered instant values in their calculations in most cases. While in a real world, the risk factors and their identifiers can be changed from one time to another for many reasons. For example, the chance of lack of money in a period (occurrence rate) could be entirely different from another period due to economic conditions. For example, Davari and Demeulemeester (2019) dealt with the proactive and reactive resource-constrained project scheduling problem with stochastic activity durations. Grabovy and Orlov (2016) developed a risk management method for considering uncertainty factors at all stages of implementing an investment construction project in Russia using a cross border index for calculating an investment construction project. Besides, the level of intensity of a machine breakdown (severity) may be varied. While

${ }^{2}$ https://dictionary.cambridge.org/dictionary/english/uncertainty- Retrieved in 11.8.2020. 
oil leakage is a minor failure in most cases, it may cause harmful damages to an engine at another time. For instance, Nasrabadi \& Mirzazadeh (2016) focused on uncertain conditions and the time value of money. In such cases, using constant values for risk identifying and risk assessments is a flawed strategy and can be useless and even in some cases, it can mislead the decision-makers. Such drawbacks are even serious for projects in the industry. In most cases, stubble cues available that show a risk level will remain at the same level until the end of the project. In fact, in most of the cases, risks can be emerged and exacerbated in a short period of a project and then they can be ameliorated by risk response programs ( $\mathrm{r}$ be worsened if they are left alone). For this purpose, in this section we focus on the MADM and MODM techniques in project management considering the uncertainty. Dempster introduced the theory of considering uncertainty in the probability of the decisionmaking process in the 1960s. Then, in 1976, Shafer published A Mathematical Theory of Evidence (Shafer, 1976). Their theory was developed based on considering the uncertainty of mathematics arrays and following fundamental but functional mathematics principles. Other scientists frequently apply their method in various fields, including engineering, management, and humanities. In continue, several important types of research that opted in Dempster-Shafer's evidence theory will be investigated.

\subsubsection{Dempster-Shafer Theory}

Using Dempster's mathematical theory of evidence can overcome the dilemma between exact methods and probabilistic methods in expert systems. Zadeh (1986) Dempster-Shafer theory of evidence theory has been widely used in A.I. for considering uncertainty in expert systems. Tang (2015) addressed a fuzzy soft set approach that worked based on grey relational analysis and Dempster-Shafer theory of evidence. Dempster-Shafer's theory of evidence was used to integrate the available alternatives into one collective alternative to choose the best alternatives. Hatefi et al. (2019) used DempsterShafer theory of evidence to develop a new model for assessing risk factors in a project associated with the environment. Their method is applied in an oil company in Iran, and the outcomes were compared with those achieved by conventional risk assessment and the fuzzy inference system methods, which showed the superiority of the proposed model in uncertain conditions of a project. Li et al. (2015) discussed that most of the previously worked methods based on the fuzzy soft sets were set based on different kinds of level soft sets, which made them too sophisticated to be investigated by decisionmakers. Therefore, they proposed a new fuzzy soft sets approach based on combining grey relational analysis with the Dempster-Shafer theory of evidence in medical diagnosis problems. In their method, Dempster-Shafer rule of evidence was used to aggregate the available alternatives into a collective alternative to select the best alternative. Wang et al. (2016) enhanced the functionality of the fuzzy soft set-based decision-making method by combining ambiguity measure and Dempster-Shafer theory of evidence, which yielded less uncertainty and increased the choice decision level accordingly. Ballent et al. (2019) believed that Dempster-Shafer's theory of evidence could provide a basis for considering various expert beliefs where structural vulnerability and damage are examined, which results in subjective assessments. Muriana \& Vizzini (2017) stated that quantitative risk assessment is an efficient tool for fast decision-making. At the same time, progress variances from what was targeted before having adverse effects on project risk profile. Thus corrective and preventive actions must be defied based on the risk index to balance the risks. Niazi et al. (2016) discussed that many software organizations do not pay enough attention to project management and risk assessment before starting global software development. For this purpose, they proposed a 2 step approach to identify and analyze the 19 risks associated with global software development from client and vendors' points of view. Pan et al. (2019) proposed a new hybrid interval-valued fuzzy sets, improved Dempster-Shafer evidence theory and fuzzy Bayesian networks for risk assessment and risk analyzing for sophisticated uncertain conditions. They showed that the proposed method could help reduce the likelihood of potential failure occurrence and ameliorate the risk magnitudes while a failure happened. Qazi et al. (2016) addressed a new method for assessing risks by considering project complexity simultaneously. They found there is interdependency available between complexity drivers, risks and objectives and their method was also able to make priority between complexity drivers, risks and strategies.

Sangaiah et al. (2018) proposed a hybrid approach for risk assessment of software projects, including fuzzy DecisionMaking Trial and Evaluation Laboratory, Fuzzy MCDM and MADM. Their method could provide more effective results compared to classic methods. Suresh \& Dillibabu (2020) focused on risk assessment of software projects using a hybrid fuzzy-based machine learning mechanism that worked based on an adaptive neuro-fuzzy inference system-based multicriteria decision-making and intuitionistic fuzzy-based TODIM approaches. Tonmoy et al. (2018) dealt with coastal risks identification and evaluation in Australia. They found that informing and consulting stakeholders has positive impacts on planning for risk management. Zou et al. (2017) stated that multi-disciplinary collaboration in risk management is necessary to achieve more success. In most of the classic risk assessment methods, risks were usually analyzed separately. However, Zhang (2016) stated that there are correlations between risk factors of a project that can influence project performance. Therefore, they proposed a new method for measuring risks interdependently, followed by an optimization model for selecting the best risk response strategies. Zavadskas et al. (2010) proposed a MADM method for risk evaluation which worked based on TOPSIS grey and COPRAS-G methods. Their main aim was to consider the stakeholders' goals along with other construction process efficiency and real estate value factors. As far as found by reviewing the papers, the following findings achieved:

1- Considering risk factors in project management is vital and during the last two decades, scientists focused on minimizing the risk factors associated with a project. 
2- Uncertainty in occurrence probability and intensity should not be ignored and will impose detrimental effects on a project. Scientists considered various aspects of uncertainty in their studies.

3- While dealing with uncertainty in risk identification and risk assessments comes into consideration, Dempster-Shafer's evidence theory is a promising way to be used.

4- In order to consider multi-attribute in evidence theory (while more than one attribute has to be addressed), evidence theory shows flexibility to combine with other decision-making methods. The hybrid methods have superiority compared to the standard decision-making methods.

5- In this research, considering the compatibility of AHP in choosing the best project and also the outstanding features of Evidence theory in addressing uncertainty, a hybrid AHP-Evidence theory will be proposed to address the problem of selecting the best industrial project among the available alternatives in order to minimize the production risks associated to a project.

The outcomes of the comprehensive research done in this section, using a hybrid AHP-Evidence theory for selecting industrial projects to minimize production risks, have not been addressed before.

\section{A Hybrid Evidence Theory and AHP}

In decision-making science, in which the choice of a solution from among the existing solutions or prioritization of decisions with multiple criteria of solutions, multi-criteria decision-making methods are considered amongst the most trustful methods. The process of hierarchical analysis reflects the natural behavior of human thinking. This technique examines complex problems based on their interactions and simply solves them. The hierarchical analysis process can be used when the decision-making action is faced with several competing options and decision criteria. The proposed criteria can be quantitative or qualitative. The basis of this decision-making method is based on pairwise comparisons. The decision-maker begins by providing a hierarchical decision tree. The decision hierarchy tree shows the factors being compared and the competing options being evaluated in the decision. Then a series of pairwise comparisons are performed. These comparisons show the weight of each factor along with the competing options evaluated in the decision process. Finally, the logic of the hierarchical analysis process combines matrices from pairwise comparisons to make the optimal decision. Let us take a deeper look at the Dempster-Shafer theory. In Dempster Shafer's theory, the level of belief of individuals in expressing their opinions is used. For example, not all survey participants necessarily answer questions with $100 \%$ certainty. Sometimes in answering a factor, they may answer with skepticism or even believe in one answer with $\alpha \%$, but they may consider another answer with less probability (let us say (1- $\alpha) \%$ ). Therefore, in this method, the degree of belief of individuals in answering each question plays a key role and is considered a function of belief. The belief function can be defined as a mathematical function, a range of values (for example, between 0 and 100) or even a quantitative and qualitative Table. Thus, DempsterShafer theory, which is often used as a method based on the degree of belief of individuals, is based on two principles: first, obtaining participants' degrees of belief for possible answers to each of the questions, and second (Dempster rule) to combine such degrees of belief when they are based on independent evidence.

\subsection{Why Hybrid Evidence Theory with AHP?}

Analytic Hierarchy Process (AHP) is a decision problem divided into different levels of objectives, criteria and sub-criteria to choose the best alternative amongst the available ones. In this process, different options are involved in decision making and it is possible to analyze the sensitivity of the criteria and sub-criteria. A sensitivity analysis that is the base of the AHP method is a way to rank alternatives in terms of the pre-defined criteria. The decision-maker can also weigh criteria. However, one major shortcoming of the classic AHP is that in this method, the values are considered constant and therefore, it cannot reflect the uncertainty of the expert's responses in a selecting problem. Therefore, considering the aim of this research, which is choosing the best project amongst the available alternatives to minimize the risks, combining this method with Evidence theory (that uses possibility, belief, and uncertainty functions) would be an excellent way to overcome this shortcoming. Reasons for choosing a Hybrid Evidence Theory and AHP are listed as following:

1) Evidence theory is a promising method for minimizing the uncertainty in decision-making problems that have been widely used before for many similar decision-making models.

2) Evidence theory can be applied to get the idea of experts and then select the best project that minimizes negative factors such as job tardiness, work in process, bottleneck machines, and over-allocated machines.

3) Coding the method is more user-friendly than mathematical modeling, especially metaheuristic models than Genetic Algorithms or other Meta-heuristics.

4) The outcomes are more understandable for project managers in real industries.

5) Hybrid Evidence theory and AHP has not been used before for "project selection problem in order to minimize the risks of implementation," and therefore, the chance of publishing the outcomes of this research will be increased (not a common, repetitive method). For example, an expert can be given a range for risk (i.e., score (1-5)) with a confidence rate of $30 \%$ and therefore, the system considers (1-9) with a confidence rate 70\% automatically. He/She can also give a single score for risk as well. For example, he/she can give 5 with a confidence rate (0.6). In this case, the algorithm considered (19) 0.5 automatically. 
Compared to the classic AHP, the proposed hybrid AHP and Dempster-Shafer Theory of Evidence have many prominent features. Table 1 outlines the features of both mentioned methods:

Table 1

Comparing Classic AHP and the Proposed Hybrid AHP and Dempster-Shafer Theory of Evidence

\begin{tabular}{|c|c|c|}
\hline Criteria & AHP & Hybrid AHP and Theory of Evidence \\
\hline Uncertainty & $x$ & $\checkmark$ \\
\hline Multi- Expert's Opinion & $x$ & $\checkmark$ \\
\hline Weight of Risks & $\checkmark$ & $\checkmark$ \\
\hline Outcome & Single Point & Risk Domain \\
\hline Speed of Solving & Fast & Moderate \\
\hline Weight of Experts' Opinions & $\times$ & $\checkmark$ \\
\hline
\end{tabular}

\subsection{Steps of the Proposed Method}

In this section, the necessary steps for designing the methodology of this project will be presented:

1. Choose The Risks Based On The Objectives Of The Projected

2. Design A Questionnaire According To The Selected Risks

•Choose The Risks Based On The Objectives Of The Projected

Fig. 2. The process of the risk assessment

\subsection{Data for Verifying the Proposed Method}

After developing the Matlab codes, it is time to evaluate the performance of the program. For this purpose, several experiments in small, medium and large-scale sizes will be solved in section 4 . The experiments are designed so that various conditions that potentially surround a company while choosing the best project would be considered. Table 2 shows data of the verification section that will be used in section 4 .

\section{Table 2}

Experiment for validation of the data

\begin{tabular}{llll}
\hline Factors & L1: Small Scale & L2: Medium Scale & L3: Large Scale \\
\hline Number of Risk Factors & $1-3$ & $4-5$ & $6-13$ \\
Number of Experts & $2-5$ & $6-10$ & $11-30$ \\
Number of Alternatives & $1-3$ & $4-5$ & $6-13$ \\
Number of contract Options & $1-2$ & $2-3$ & $4-5$ \\
\hline
\end{tabular}

Design of Experiments (DOE) will be used for designing the exact number of experiments of parameters. In this regard, a Taguchi design will be developed using Minitab 18. 


\subsection{Dependent and Independent Variables}

In this research, the significant risk factors that can influence a project's success must be found first. Therefore, "Project Success" can be defined as the dependent variable. According to the questions of the research, the risk factors that can impose detrimental effects on the success of a project are categorized into the four main sections:

- $\quad$ Properties Risk Factors (Infrastructure, Machinery, Human Resource)

- Technology and Operational Risk Factors (Scheduling, Technology, Operational Risk, Management Systems)

- Financial Risk Factors (Evaluating projects, Profit and Costs, Money Value)

- Strategic Risk Factor (Competition, Market share, Marketing, Customer Satisfaction)

This research aims to find out if the above risk categories can influence the success of a project. If so, to what extent? Therefore, the following variables are to be addressed in this research:

- Dependent Variable: Project Success

- Independent Variables: Table 3 indicates the independent variables according to their risk factor categories.

Since in this research, one aim is to track the influence of a variable throughout the life cycle of a project, each of the above variables will be asked in 3 phases:

4 Before selecting a project

5 During the execution

6 After finishing the project

Using this strategy, finding the correlations between the independent variables and dependent variables can show us if a project is selected correctly or not. Moreover, more importantly, does study companies pay enough attention to such risk factors?

Table 3

Risk Factor Categories and Related Independent Variables

\begin{tabular}{|c|c|c|c|c|c|}
\hline \multirow{2}{*}{$\begin{array}{c}\text { Risk Factor } \\
\text { Category }\end{array}$} & \multirow{2}{*}{ No. } & \multirow{2}{*}{ Variable (Risk Factor) } & \multicolumn{3}{|c|}{ Questions } \\
\hline & & & Before Project Selection & During Execution & After Project \\
\hline \multirow{3}{*}{ Properties } & 1 & Infrastructures & Q1 & Q4 & Q7 \\
\hline & 2 & Machinery & Q2 & Q5 & Q8 \\
\hline & 3 & Human Resource & Q3 & Q6 & Q9 \\
\hline \multirow{5}{*}{$\begin{array}{c}\text { Technologic and } \\
\text { Operational }\end{array}$} & 4 & Project Scheduling Performance & Q10 & Q15 & Q20 \\
\hline & 5 & Project Schedule (Time and Cost) & Q11 & Q16 & Q21 \\
\hline & 6 & Required Technology & Q12 & Q17 & Q22 \\
\hline & 7 & Risk Management & Q13 & Q18 & Q23 \\
\hline & 8 & Management systems & Q14 & Q19 & Q24 \\
\hline \multirow{3}{*}{ Financial } & 9 & Tender Process Performance & Q25 & Q28 & Q31 \\
\hline & 10 & Costs And Profits Estimation & Q26 & Q29 & Q32 \\
\hline & 11 & Money Value & Q27 & Q30 & Q33 \\
\hline \multirow{4}{*}{ Strategic } & 12 & Rivals' Strategies & Q34 & Q38 & Q42 \\
\hline & 13 & Market Share Prediction & Q35 & Q39 & Q43 \\
\hline & 14 & Business Plan & Q36 & Q40 & Q44 \\
\hline & 15 & Customer Satisfaction & Q37 & Q41 & Q45 \\
\hline
\end{tabular}

\section{A Hybrid AHP and Evidence Theory by Considering Significant Risk Factors}

\section{- Solution Representing}

The solutions of the proposed hybrid AHP and Dempster-Shafer theory of evidence can be represented as follows:

- Total_Risk_Matrix_of_Project(Number_of_Contract_options,2,Number_of_Projects)

where the first index of the above matrix indicates several available Contract options, the second index (2) is used for showing the upper and the lower level of risk of each alternative (upper-risk level, lower risk level); and the $3^{\text {rd }}$ index is used to show the number of alternatives. The above matrix will indicate the risk levels of each alternative. Therefore, using the statistical probability method, as shown below, the best alternative, which contains the lowest risk domain, can be elected using the following formula:

- A.I. $=[\ldots]$ 
The (Alternative Index) A.I. matrix shows the amount of $\frac{1}{\mu_{i}}$ that is the index for showing the mean of total risk factor values of an alternative. The greater values of A.I. are preferred. Afterward, the best Alternatives can be detected and represented.

\section{- Choosing an Alternative with Lowest Risk Domain}

After calculating the total value for risk factors as a domain (with upper and lower limits) for each alternative, it is time to select the alternative with the lowest total risk factor. However, choosing the best alternative in this research is not as easy as selecting the project with the lowest value; because here, the total risk values are not exact numbers, and it is not possible to easily select the minimum value. To overcome such a problem, two main factors must be considered:

- Mean of the total risk factor domain $\left(\mu_{i}\right)$

- The length of the total risk factor domain $\left(d_{i}\right)$

It is obvious that an alternative with the lowest $\mu_{i}$ is more desired as it means that the related project achieved the lowest risk values (Fig. 3). However, while the $\mu_{i}$ for 2 alternatives are the same, the project with smaller $d_{i}$ is preferred because, generally, it has a lower risk than the other option (Fig. 4).

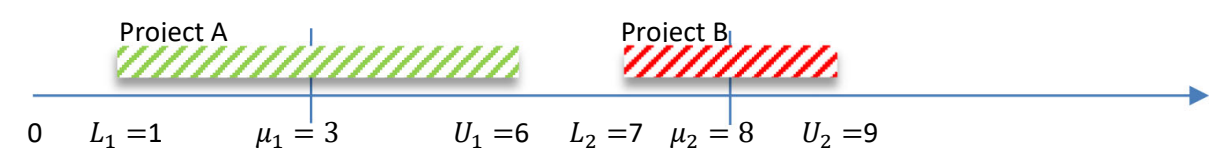

Fig. 1. The project with the lowest $\boldsymbol{\mu}_{\boldsymbol{i}}$ is more preferred

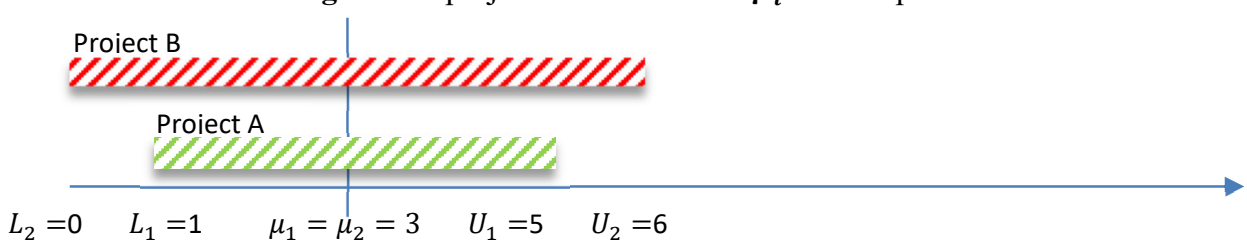

Fig. 2. While $\boldsymbol{\mu}_{\boldsymbol{i}}$ is equal for 2 alternatives, the alternative with lower $\boldsymbol{\sigma}_{\boldsymbol{i}}$ is more preferred

One crucial point is that since according to the central limit theorem, the mean of the variables follows the Normal distribution function, then the following statements are factual (with $P=0.997$ ):

$$
\begin{aligned}
& U_{i} \cong \mu_{i}+3 \sigma_{i} \\
& L_{i} \cong \mu_{i}-3 \sigma_{i} \\
& U_{i}-L_{i}=6 \sigma_{i}
\end{aligned}
$$

Therefore, the index $\frac{6 \sigma_{i}}{\mu_{i}}$ can be considered as an appropriate index for comparing the total risk domains while two alternatives have equal means $\left(\mu_{i}=\mu_{i}^{\prime}\right)$.

$$
C . V=\frac{6 \sigma_{i}}{\mu_{i}}
$$

In statistics, $\frac{\sigma_{i}}{\mu_{i}}$ is usually used instead of the $\frac{6 \sigma_{i}}{\mu_{i}}$. Therefore, all possible conditions must be taken into account.

1) While 2 alternatives have different total risk factor means $\left(\mu_{i} \neq \mu_{j}\right)$ and different total risk factor domain lengths $\left(d_{i} \neq d_{j}\right)$, as shown by Fig. 5 .

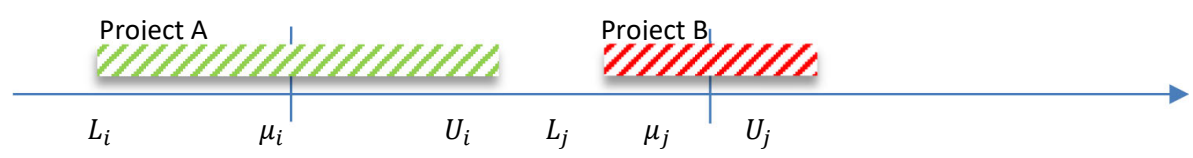

Fig. 5. Possible Condition 1- Different Means and Different Domain Length

$\frac{1}{\mu_{i}} \neq \frac{1}{\mu_{j}} \quad \& \quad \frac{\sigma_{i}}{\mu_{i}} \neq \frac{\sigma_{j}}{\mu_{j}}$ 
Result: Project with lower $\mu_{i}$ will be selected (more significant $A . I=\frac{1}{\mu_{i}}$ ).

2) While two alternatives have different total risk factor means $\left(\mu_{i} \neq \mu_{j}\right)$ but equal total risk factor domain lengths $\left(d_{i}=\right.$ $d_{j}$ ), as shown by Fig. 6 .

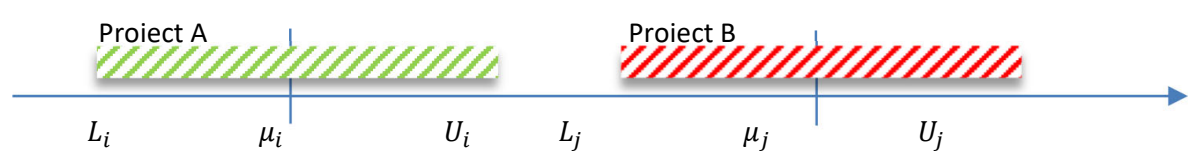

Fig. 6. Possible Condition 1- Different Means and Similar Domain Length

$\frac{1}{\mu_{i}} \neq \frac{1}{\mu_{j}} \& \frac{\sigma_{i}}{\mu_{i}}=\frac{\sigma_{j}}{\mu_{j}}$

A. $I_{i} \neq A . I_{j} \quad \& C . V_{i}=C . V_{j}$

Result: Project with lower $\mu_{i}$ will be selected (more significant $A . I=\frac{1}{\mu}$ ).

3) While two alternatives have equal total risk factor means $\left(\mu_{i}=\mu_{j}\right)$ but different total risk factor domains $\left(d_{i} \neq d_{j}\right)$, as shown by Fig. 7 .

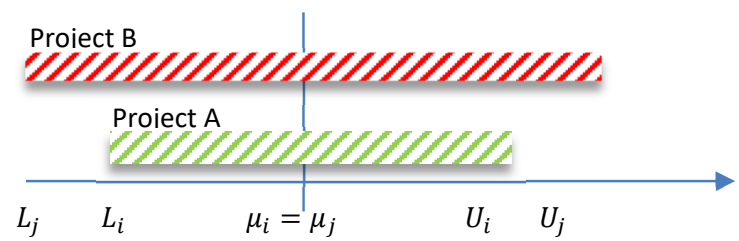

Fig. 7. Possible Condition 1- Similar Means and Different Domain Length

$\frac{1}{\mu_{i}}=\frac{1}{\mu_{j}} \& \frac{\sigma_{i}}{\mu_{i}}=\frac{\sigma_{j}}{\mu_{j}}$
A. $I_{i}=A . I_{j} \quad \& C . V_{i}=C . V_{j}$

Result: Projects have the same A.I; therefore, the project with lower C.V is preferred.

3) While two alternatives have equal total risk factor means $\left(\mu_{i}=\mu_{j}\right)$ and equal total risk factor domains $\left(d_{i}=d_{j}\right)$, as shown by Fig. 8 .

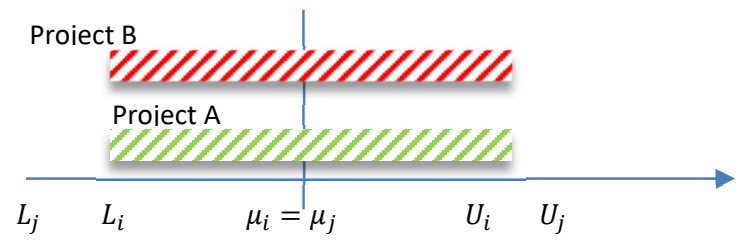

Fig. 8. Possible Condition 1- Similar Means and Similar Domain Length

$\frac{1}{\mu_{i}}=\frac{1}{\mu_{j}} \quad \& \quad \frac{\sigma_{i}}{\mu_{i}}=\frac{\sigma_{j}}{\mu_{j}}$

$A . I_{i}=A . I_{j} \quad \& C . V_{i}=C . V_{j}$

Result: Projects has the same A.I and C.V. Both Alternatives can be chosen.

\section{Results and discussion}

5.1 Verifying the Proposed Algorithm (Solving experiments gathered from the literature) 
In this section, using an L2^4 Taguchi method for DOE using Minitab 18.0 is used. The reason for choosing this type is that a lower limit and an upper limit are considered $\left(2^{\wedge} 4\right)$. Several case studies will be solved to verify the functionality of the proposed algorithm in different ways. The case studies are designed in a way that various range of parameters are taken into account. Therefore, the case studies are divided into three main categories used by many researchers in the literature review (Delgoshaei \& Gomes, 2016). Table 5 to Table 7 show the case studies that the proposed algorithm must perform. As seen, the domains of each of the case studies have been selected according to Table 4 to cover each scale (small, medium, large, and very large) entirely.

Table 1

The Designed Case Studies (Taguchi L2^4)

\begin{tabular}{|c|c|c|c|c|c|}
\hline No. & Scale & Number of Experts & Number of Risk Factors & Number of Alternatives & Number of Contract Options \\
\hline 1 & \multirow{8}{*}{ 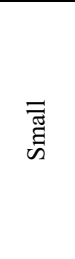 } & 2 & 1 & 2 & 1 \\
\hline 2 & & 2 & 1 & 3 & 2 \\
\hline 3 & & 2 & 3 & 2 & 2 \\
\hline 4 & & 2 & 3 & 3 & 1 \\
\hline 5 & & 5 & 1 & 2 & 2 \\
\hline 6 & & 5 & 1 & 3 & 1 \\
\hline 7 & & 5 & 3 & 2 & 1 \\
\hline 8 & & 5 & 3 & 3 & 2 \\
\hline 9 & \multirow{8}{*}{ 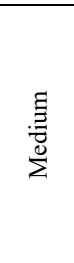 } & 6 & 4 & 4 & 2 \\
\hline 10 & & 6 & 4 & 5 & 3 \\
\hline 11 & & 6 & 5 & 4 & 3 \\
\hline 12 & & 6 & 5 & 5 & 2 \\
\hline 13 & & 10 & 4 & 4 & 3 \\
\hline 14 & & 10 & 4 & 5 & 2 \\
\hline 15 & & 10 & 5 & 4 & 2 \\
\hline 16 & & 10 & 5 & 5 & 3 \\
\hline 17 & \multirow{8}{*}{$\begin{array}{l}\stackrel{\mathscr{D}}{0} \\
\stackrel{0}{\Xi} \\
ت\end{array}$} & 11 & 6 & 6 & 4 \\
\hline 18 & & 11 & 6 & 10 & 5 \\
\hline 19 & & 11 & 13 & 6 & 5 \\
\hline 20 & & 11 & 13 & 10 & 5 \\
\hline 21 & & 30 & 6 & 6 & 5 \\
\hline 22 & & 30 & 6 & 10 & 4 \\
\hline 23 & & 30 & 13 & 6 & 5 \\
\hline 24 & & 30 & 13 & 10 & 5 \\
\hline
\end{tabular}

In this section, each of the case studies will be solved by the proposed algorithm in Matlab. The outcomes of the case studies are shown in Table 5. However, in order to see the steps of the proposed algorithm, in reality, case study number 2 in Table 5 will be explained in detail.

Table 2

Results of Solving Numerical Experiments Using the Proposed Hybrid AHP and Theory of Evidence Algorithm

\begin{tabular}{|c|c|c|c|c|c|c|c|c|c|c|c|}
\hline No. & Scale & $\begin{array}{c}\text { Number of } \\
\text { Experts }\end{array}$ & $\begin{array}{c}\text { Number of } \\
\text { Risk Factors }\end{array}$ & $\begin{array}{l}\text { Number of Al- } \\
\text { ternatives }\end{array}$ & $\begin{array}{l}\text { Number of Con- } \\
\text { tract Options }\end{array}$ & Solving Time & $\begin{array}{l}\text { Best Al- } \\
\text { ternative }\end{array}$ & $\begin{array}{c}\text { Best } \\
\text { Contract Option }\end{array}$ & $\begin{array}{r}\text { Minimum } \\
\text { Risk D } \\
\end{array}$ & $\begin{array}{l}\text { Observed } \\
\text { omain }\end{array}$ & Observed AI \\
\hline 1 & \multirow{8}{*}{ 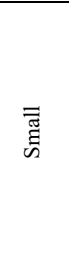 } & 2 & 1 & 2 & 1 & 0.036 & 1 & 1 & {$[0.7622$} & $1.3121]$ & 0.9642 \\
\hline 2 & & 2 & 1 & 3 & 2 & 0.041 & 2 & 2 & {$[0.0447$} & $1.0356]$ & 1.8514 \\
\hline 3 & & 2 & 3 & 2 & 2 & 0.054 & 2 & 1 & {$[0.1675$} & $2.5457]$ & 0.7371 \\
\hline 4 & & 2 & 3 & 3 & 1 & 0.041 & 2 & 1 & {$[0.5039$} & 3.4899] & 0.5008 \\
\hline 5 & & 5 & 1 & 2 & 2 & 0.037 & 2 & 1 & {$[0.1605$} & $1.6070]$ & 1.1315 \\
\hline 6 & & 5 & 1 & 3 & 1 & 0.047 & 3 & 1 & {$[0.4416$} & $2.2646]$ & 0.7391 \\
\hline 7 & & 5 & 3 & 2 & 1 & 0.039 & 1 & 1 & {$[0.3602$} & 3.1599] & 0.5682 \\
\hline 8 & & 5 & 3 & 3 & 2 & 0.054 & 2 & 2 & {$[0.1753$} & $1.5428]$ & 1.1641 \\
\hline 9 & \multirow{8}{*}{ 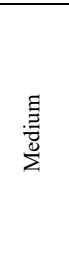 } & 6 & 4 & 4 & 2 & 0.034 & 4 & 1 & {$[0.2607$} & $1.3144]$ & 1.2698 \\
\hline 10 & & 6 & 4 & 5 & 3 & 0.084 & 5 & 1 & {$[0.1204$} & 1.1896] & 1.5267 \\
\hline 11 & & 6 & 5 & 4 & 3 & 0.088 & 1 & 3 & {$[0.1021$} & $1.4210]$ & 1.3353 \\
\hline 12 & & 6 & 5 & 5 & 2 & 0.049 & 3 & 1 & {$[0.1982$} & $1.8828]$ & 0.9610 \\
\hline 13 & & 10 & 4 & 4 & 3 & 0.044 & 3 & 3 & {$[0.0331$} & $3.3072]$ & 0.5987 \\
\hline 14 & & 10 & 4 & 5 & 2 & 0.033 & 1 & 2 & {$[0.0585$} & 4.4972] & 0.4390 \\
\hline 15 & & 10 & 5 & 4 & 2 & 0.039 & 3 & 2 & {$[0.0652$} & $4.1237]$ & 0.4775 \\
\hline 16 & & 10 & 5 & 5 & 3 & 0.082 & 1 & 3 & {$[0.0464$} & 2.5759] & 0.7627 \\
\hline 17 & \multirow{8}{*}{$\begin{array}{l}\stackrel{.0}{.0} \\
\text { 哥 }\end{array}$} & 11 & 6 & 6 & 4 & 0.186 & 3 & 4 & {$[0.0509$} & $1.3212]$ & 1.4577 \\
\hline 18 & & 11 & 6 & 10 & 5 & 0.557 & 7 & 1 & {$[0.0366$} & $1.1134]$ & 1.7391 \\
\hline 19 & & 11 & 13 & 6 & 5 & 0.144 & 1 & 5 & {$[0.0365$} & $1.2240]$ & 1.5867 \\
\hline 20 & & 11 & 13 & 10 & 5 & 0.200 & 1 & 5 & {$[0.0365$} & $1.2240]$ & 1.5867 \\
\hline 21 & & 30 & 6 & 6 & 5 & 0.062 & 2 & 2 & {$[0.0327$} & $1.2286]$ & 1.5857 \\
\hline 22 & & 30 & 6 & 10 & 4 & 0.08 & 3 & 4 & {$[0.0393$} & $1.5342]$ & 1.2710 \\
\hline 23 & & 30 & 13 & 6 & 5 & 0.109 & 1 & 4 & {$[0.0320$} & $1.2514]$ & 1.5584 \\
\hline 24 & & 30 & 13 & 10 & 5 & 0.191 & 10 & 4 & {$[0.0319$} & $1.2293]$ & 1.5858 \\
\hline
\end{tabular}


- Solving a case study and explaining the outcomes in detail

In this section and to explain the algorithm functionality's mechanism, the $3^{\text {rd }}$ case study in Table 5 will be explained in detail. Suppose a company has to select the best option between the available 2 alternatives. One is to set up a new production line and the other is to set up a new laboratory, which can provide outdoor services as well.

- Number_of_Alternatives $=2$

There are 2 managers in this company that must determine which alternative to be carried out in future. However, the quote of the company share for the first manager is 2 times more than the other manager, and therefore his vote will value 2 times more than the other.

- Number_of_Experts $=2$

- Weight_of_experts=[0.3 0.7]

In order to choose the best option, managers decided to consider 3 risk factors: financial risk factor, operational risk factor, and property risk factor. From the manager's point of view, at this time, the financial risk factor is more important than the other factors, and the operational risk factors are more important than the properties risk factor. Therefore, they decided to set the following values between the risk factors:

- Number_of_Risks=3

- Weight_of_Risks=[0.5 0.30 .2$]$

Regardless of the project title, the company has 2 options for financing it. One is to pay the expenditures directly and the other is to get a bank loan. However, each contract option will influence the level of the risks.

- Number_of_Contract_options=2

Afterward, the managers are asked to fill out a questionnaire to set values about each risk factor and to set their uncertainty about their solutions. The following matrix shows the first 3 experts' opinions (Table 6):

Table 3

Expert Opinion Total Matrix

\begin{tabular}{ccc}
$\begin{array}{c}\text { Expert_Total_Matrix } \\
\text { (Expert 1) }\end{array}$ & $\begin{array}{c}\text { Expert_Total_Matrix } \\
\text { (Expert 2) }\end{array}$ & $\begin{array}{c}\text { Expert_Total_Matrix } \\
\text { (Expert 3) }\end{array}$ \\
\hline $\mathbf{4 4 3 0 . 1 ;}$ & {$[240.1 ;$} & {$[780.9 ;$} \\
$\mathbf{8 ~ 8 ~ 0 . 2 ] ;}$ & $880.2] ;$ & $550.9] ;$ \\
\hline
\end{tabular}

After solving the case study using the proposed hybrid AHP and Theory of Evidence, the following results are obtained.

$>$ Step 1) Calculating the "Expected_value_for_risk" matrix using the experts' opinions (Table 7):

Table 4

Expected Value of Risks

\begin{tabular}{rrrr} 
Expected_value_for_risk 1 & Expected_value_for_risk 2 \\
\hline $\mathbf{3 . 6 6 6 7}$ & $\mathbf{3 . 3 0 0 0}$ & 1.2333 & 5.7333 \\
$\mathbf{1 . 1 6 6 7}$ & $\mathbf{5 . 7 6 6 7}$ & 1.6000 & 5.6000 \\
$\mathbf{3 . 6 6 6 7}$ & $\mathbf{4 . 5 0 0 0}$ & 0.8667 & 5.3333
\end{tabular}

The way for calculating the $1^{\text {st }}$ element of the above matrix will be explained:

In order to calculate the above matrix, the lower and upper value for each risk factors must be calculated. Therefore, using the "for" loop, the idea for each expert will be gained. For example, for the first risk factor the results will be as follows (Table 8):

Table 5

Upper and Lower Limit of Expected Value for Risks

Lower limit of Expected value for risk

Upper limit of Expected value for risk

$\begin{array}{ccccccc}\mathbf{6 . 4 0 0 0} & \mathbf{0} & \mathbf{0} & 4.5000 & 0 & 0 \\ 11.0000 & \mathbf{0} & \mathbf{0} & 9.9000 & 0 & 0\end{array}$

Using the same strategy, the rest elements of the "Expected value for risk" will be calculated (Table 9). 
Table 6

Expected Value for Risks

Expected value for risk(:,:,1)

Expected value for $\operatorname{risk}(:,:, 2)$

$\begin{array}{llll}\mathbf{3 . 6 6 6 7} & \mathbf{3 . 3 0 0 0} & 1.2333 & 5.7333 \\ \mathbf{1 . 1 6 6 7} & \mathbf{5 . 7 6 6 7} & 1.6000 & 5.6000 \\ \mathbf{3 . 6 6 6 7} & \mathbf{4 . 5 0 0 0} & 0.8667 & 5.3333\end{array}$

$>$ Step 2) In continuation, the mean of the will be calculated for each of the risks in each contract option (Table 10)

Table 7

Risk Associate Matrix

\begin{tabular}{ccccc}
\hline Risk & Lower Risk Associate Matrix & Upper Risk Associate Matrix \\
\hline \multirow{2}{*}{ Risk 1 } & 0.9000 & 0.5756 & 1.1111 & 2.9730 \\
\multirow{2}{*}{ Risk 2 } & 0.3364 & 0.2151 & 1.7374 & 4.6486 \\
& 0.2023 & 0.2083 & 4.9429 & 3.6042 \\
Risk 3 & 0.2775 & 0.2857 & 4.8000 & 3.5000 \\
& 0.8148 & 0.6875 & 1.2273 & 5.1923 \\
\end{tabular}

$>$ Step 4) Normalize the Upper and lower risk factor values (Table 11)

Table 8

Normalized Risk Factor Matrix

Normalized_Upper_Risk_Associate_Matrix(:,:,2) Normalized_Upper_Risk_Associate_Matrix(:,:,3)

$\begin{array}{llll}\mathbf{1 0 . 3 0 2 6} & \mathbf{7 . 2 9 5 2} & 1.2182 & 6.1086 \\ \mathbf{1 0 . 0 0 4 8} & \mathbf{7 . 0 8 4 3} & 1.4439 & 7.2398\end{array}$

$>$ Step 5) Calculating the average of the normalized risk matrix:

Then, using the following formulas, the average of the normalized lower and upper-risk factors will be calculated (Table 12).

Table 9

Averaged Normalized Risk Factor

\begin{tabular}{ccc}
\hline \multirow{2}{*}{ Risk } & $\begin{array}{c}\text { Averaged Normalized Lower Risk Asso- } \\
\text { ciate Matrix }\end{array}$ & $\begin{array}{c}\text { Averaged Normalized Upper Risk Associ- } \\
\text { ate Matrix }\end{array}$ \\
\multirow{2}{*}{ Risk 1 } & 0.1957 & 2.3293 \\
& 0.0732 & 3.6422 \\
Risk 2 & 0.0250 & 8.7989 \\
& 0.0343 & 8.5446 \\
Risk 3 & 0.1822 & 3.6634 \\
& 0.0431 & 4.3418 \\
\hline
\end{tabular}

Step 6) Calculating the Total Risk matrix:

In the next step, the total lower and upper risk matrix will be calculated (Table 13).

Table 10

Total Risk Matrix

Total Lower Risk Matrix

$\begin{array}{llll}0.0979 & 0.0075 & 0.0364\end{array}$

$0.0366 \quad 0.0103 \quad 0.0086$
Total Upper Risk Matrix

$\begin{array}{lll}1.1647 & 2.6397 & 0.7327\end{array}$

$\begin{array}{lll}1.8211 & 2.5634 & 0.8684\end{array}$

At this point, the total lower and upper values for each alternative (using a specific contract option) are calculated. For example, for the $1^{\text {st }}$ alternative, while it is assumed to be carried out by the $2^{\text {nd }}$ contract option, the total risk domain will be [0.0366, 1.8211]. 
$>$ Step 7: Calculating the A.I. matrix and choosing the best alternative

Now and in the last step, the alternative with the lowest risk point must be selected. However, since the risk point is not an exact value but a domain, selecting the project with the lowest risk domain is not easy. To solve this problem, two factors must be taken into consideration:

- The mean of a risk factor domain $\left(\mu_{i}\right)$ where the index $A I=\frac{1}{\mu_{i}}$ is used for it as described in section 3 .

- If 2 or more projects have the same $\mu_{i}$, then the length of the risk factor domain where $\sigma_{i}$ is used for it (using the $C V=\frac{\sigma_{i}}{\mu_{i}}$ formula) as described in section 3 .

Therefore, using the following formulas, the A.I. index will be calculated for each alternative.

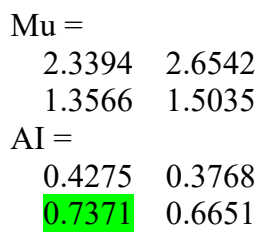

Then, the best option will be the alternative with the highest A.I. index value, which in this case study is the $2^{\text {nd }}$ project while the $1^{\text {st }}$ contract option is selected for it $(0.7371)$.

- $\quad$ Best_Alternative $=2$

- Best_ContractOption $=1$

\subsection{Measuring the Performance of the Proposed Algorithm}

In order to assess the performance of the proposed method, several indicators are defined as shown below:

- The ability to solve all problem types

- The ability to choose projects with the lowest uncertainty

- The solving time

- Comparing the hybrid AHP and Dempster-Shafer Theory of Evidence with Classic AHP

In addition, in the second part of this section, the outcomes of solved problems using the hybrid AHP and Dempster-Shafer Theory will be compared with classic AHP to show the superiority of the proposed method in solving the problems while uncertainty has existed.

- The ability to solve all problem types

The results of solving 24 experiments solved by the proposed hybrid method showed that the algorithm could solve all experiments $(100 \%)$ and show the best alternative with the lowest risk factors' average.

$$
\text { Validating. Index }=\frac{\text { Number of Solved Cases }}{\text { Number of Designed Cases }} \cdot 100=\frac{24}{24} \cdot 100=100 \%
$$

Therefore, the algorithm can be used in real project selection time in industries.

- The ability to choose projects with the lowest uncertainty

The outcomes of all solved case studies are revised again. In each case, the A.I. matrix is presented in Table 14 and the lowest risk factor reported by the proposed algorithm is double-checked. In all studied cases, the solving algorithm is capable of finding and reporting the project with the lowest uncertainty (highest A.I.).

$$
\text { Reduced Risk Indicator }=\frac{(\text { Max A.I-Min A.I })}{\operatorname{Max} \text { A.I }} .100
$$

RRI\% shows how much percentage using the proposed algorithm helps select the alternative with the lowest risk factor. 
Table 11

The results of RPI\% for studies while solved by the proposed method

\begin{tabular}{cccccc}
\hline Row & $\begin{array}{c}\text { Reported AI value by the } \\
\text { algorithm }\end{array}$ & R.R.I\% & Row & $\begin{array}{c}\text { Reported AI value by } \\
\text { the algorithm }\end{array}$ & R.R.I\% \\
\hline 1 & 0.9642 & $90.53 \%$ & 13 & 0.5987 & $5.66 \%$ \\
2 & 1.8514 & $28.92 \%$ & 14 & 0.4390 & $58.79 \%$ \\
3 & 0.7371 & $48.88 \%$ & 15 & 0.4775 & $12.13 \%$ \\
4 & 0.5008 & $70.01 \%$ & 16 & 0.7627 & $20.57 \%$ \\
5 & 1.1315 & $48.02 \%$ & 17 & 1.4577 & $19.61 \%$ \\
6 & 0.7391 & $48.63 \%$ & 18 & 1.7391 & $12.31 \%$ \\
7 & 0.5682 & $3.41 \%$ & 19 & 1.5867 & $9.67 \%$ \\
8 & 1.1641 & $37.38 \%$ & 20 & 1.5867 & $9.67 \%$ \\
9 & 1.2698 & $44.70 \%$ & 21 & 1.5857 & $4.48 \%$ \\
10 & 1.5267 & $25.27 \%$ & 22 & 1.2710 & $10.24 \%$ \\
11 & 1.3353 & $94.45 \%$ & 23 & 1.5584 & $1.76 \%$ \\
\hline
\end{tabular}

As seen in Fig. 10, the algorithm can choose the alternative with the lowest risk value in all cases.

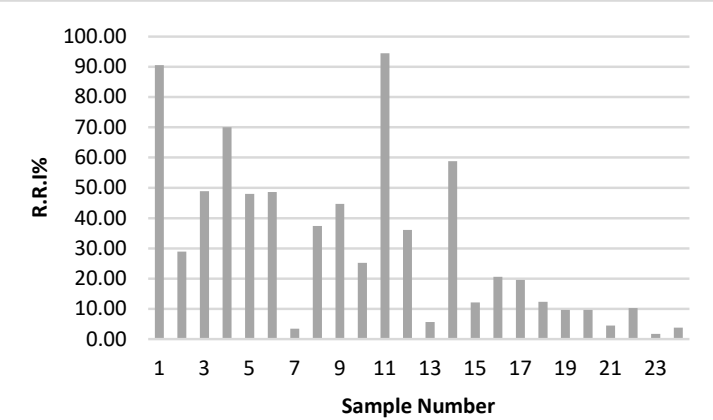

Fig. 3. The results of the R.R.I\% index for the solved case studies

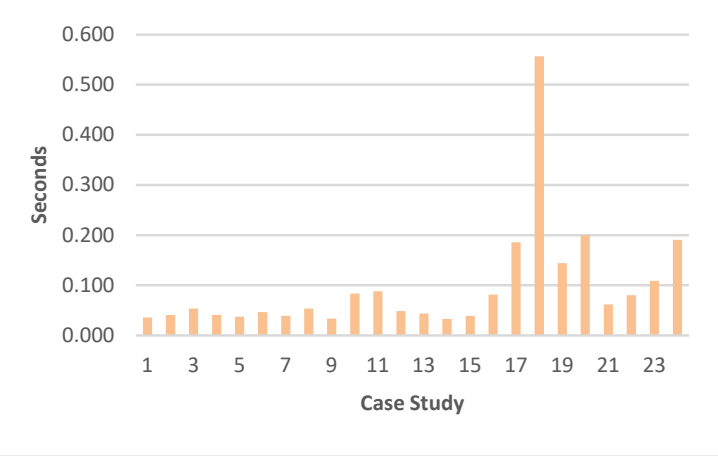

Fig. 4. The results of solving time for the studied cases

As seen, the solving algorithm solved the small case studies in less than 0.055 seconds, medium scale cases in less than 0.09 seconds, and large-scale cases in less than 0.56 seconds (Fig. 11). These results are noticeable and mean that the algorithm can be used safely in actual practice.

\section{Conclusion and recommendations}

This research focused on the uncertainty in the industrial project selection problem. In the real environment, several risk factors threaten the project's success. However, such risk factors are not constant and may take various values depending on the environment surrounding a project. Therefore, the classic decision-making methods may fail to correctly report the actual risk factors value and select the best project among the alternatives. In this research, many risk factors that influence project success are extracted using the Delphi method. The findings showed that the risks could be divided into 4 main risk clusters, which are: Properties Risk Factors; Technology and Operational Risk Factors; Financial Risk Factors and Strategic Risk Factors. In each of the risk factor clusters, several variables are defined. Each variable is asked in 3 phases of a project, which are before selecting a project, during execution of the project, and after completing the project. The aim was to track the status of a variable in the life cycle of a project. For each question, after asking the responder's opinion, his belief rate was also asked to clarify the uncertainty of the risk factors. The statistical analysis is then carried out to specify the variable's statistical description, find out the correlations between the variables and determine their values in project success (as the dependent variable). A new hybrid AHP and Demspter-Shafer Theory of Evidence are proposed, which were worked based on the uncertainty level of the risk factors. The proposed method could determine the total risk level range for each alternative and then report the best alternative with the lowest total risk level range. Then, a Taguchi Method (L2^4) is designed for designing the experiments. The proposed method is used to solve 24 experiments where the condition of the experiments was different from one experiment to another. The performance of the proposed algorithm is then evaluated using 4 indicators. The proposed method could solve all small, medium and large-scale experiments (Validating Index). Moreover, it could find and report the project with the lowest total risk range in all cases. In order to check the performance of the proposed method in choosing projects with the lowest total risk factor, the maximum and minimum risk factors for available 
alternatives of each case study are compared (Reduced Risk Indicator). The outcomes showed that the proposed hybrid method could select projects with the lowest total risk factor of up to $90.53 \%$ for small-scale studied cases, up to $94.45 \%$ for medium-scale studied cases and $19.61 \%$ for large-scale studied cases the scale of the case studies. The proposed method solved the Small scale problems in [0.036 0.054], Medium scale problems in [0.033 0.088], and Large-scale problems in [0.062 0.557] seconds depending on the nature of the Project (Processing time).

It is recommended to develop a Java application for the proposed method in this research. This could be done by computer science researchers or manufacturing engineering researchers familiar with programming languages. It is also suggested to use different MADAM methods such as VICOR and TOPSIS and compare the functionality of the proposed method in this research with them.

\section{Acknowledgments}

Authors would like to thank the anonymous reviewers and the editor for their efforts during the publication process.

\section{References}

Abd El-Karim, M. S. B. A., Mosa El Nawawy, O. A., \& Abdel-Alim, A. M. (2017). Identification and assessment of risk factors affecting construction projects. HBRC Journal, 13(2), 202-216.

Ansarifar, J., Tavakkoli-Moghaddam, R., Akhavizadegan, F., \& Hassanzadeh Amin, S. (2018) Multi-objective integrated planning and scheduling model for operating rooms under uncertainty. Proceedings of the Institution of Mechanical Engineers, Part H: Journal of Engineering in Medicine, 232(9), 930-948.

Абдикеев, Н. М., Богачев, Ю. С., \& Бекулова, С. Р. (2019). Инвестиционный потенциал обрабатывающей промышленности. Финансы: Теория и Практика, 23(4).

Ballent, W., Corotis, R. B., \& Torres-Machi, C. (2019). Representing uncertainty in natural hazard risk assessment with Dempster Shafer (Evidence) theory. Sustainable and Resilient Infrastructure, 4(4), 137-151.

Bowers, J., \& Khorakian, A. (2014). Integrating risk management in the innovation project. European Journal of innovation management, 17(1), 25-40.

Brustbauer, J. (2016). Enterprise risk management in SMEs: Towards a structural model. International Small Business Journal, 34(1), 70-85.

Carvalho, M. M. d., \& Rabechini Junior, R. (2015). Impact of risk management on project performance: the importance of soft skills. International Journal of Production Research, 53(2), 321-340.

Chemweno, P., Pintelon, L., Van Horenbeek, A., \& Muchiri, P. (2015). Development of a risk assessment selection methodology for asset maintenance decision making: An analytic network process (ANP) approach. International Journal of Production Economics, 170, 663-676.

Dašić, B., Trklja, R., \& Savić, M. (2019). Comparative analysis of inflows and sectoral distribution of the foreign direct investments flows. Mining and Metallurgy Engineering Bor(1-2), 31-40.

Davari, M., \& Demeulemeester, E. (2019). The proactive and reactive resource-constrained project scheduling problem. Journal of Scheduling, 22(2), 211-237.

DELGOSHAEI, A. (2016). SCHEDULING DYNAMIC CELLULAR MANUFACTURING SYSTEMS IN THE PRESENCE OF COST UNCERTAINTY USING HEURISTIC METHOD.

Delgoshaei, A., \& Gomes, C. (2016). A multi-layer perceptron for scheduling cellular manufacturing systems in the presence of unreliable machines and uncertain cost. Applied Soft Computing, 49, 27-55.

Delgoshaei, A., Rabczuk, T., Ali, A., \& Ariffin, M. K. A. (2017). An applicable method for modifying over-allocated multimode resource constraint schedules in the presence of preemptive resources. Annals of Operations Research, 259(1-2), 85-117.

Dziadosz, A., \& Rejment, M. (2015). Risk analysis in construction project-chosen methods. Procedia Engineering, 122, 258-265.

Esmaeili, B., Hallowell, M. R., \& Rajagopalan, B. (2015a). Attribute-based safety risk assessment. I: Analysis at the fundamental level. Journal of Construction Engineering and Management, 141(8), 04015021

Esmaeili, B., Hallowell, M. R., \& Rajagopalan, B. (2015b) Attribute-based safety risk assessment. II: Predicting safety outcomes using generalized linear models. Journal of Construction Engineering and Management, 141(8), 04015022.

Fabricius, G., \& Büttgen, M. (2015). Project managers' overconfidence: how is risk reflected in anticipated project success? Business Research, 8(2), 239-263.

Fang, C., Marle, F., \& Xie, M. (2016). Applying importance measures to risk analysis in engineering project using a risk network model. IEEE Systems Journal, 11(3), 1548-1556.

Grabovy, P., \& Orlov, A. (2016). The overall risk assessment and management: implementation of foreign investment construction megaprojects by Russian development companies. Procedia Engineering, 153, 195-202.

Gutjahr, W. J. (2015). Bi-objective multi-mode project scheduling under risk aversion. European Journal of Operational Research, 246(2), 421-434.

Hatefi, S. M., Basiri, M. E., \& Tamošaitienė, J. (2019). An evidential model for environmental risk assessment in projects using dempster-shafer theory of evidence. Sustainability, 11(22), 6329. 
Hossen, M. M., Kang, S., \& Kim, J. (2015). Construction schedule delay risk assessment by using combined AHP-RII methodology for an international NPP project. Nuclear Engineering and Technology, 47(3), 362-379.

Islam, M. S., Nepal, M. P., Skitmore, M., \& Attarzadeh, M. (2017). Current research trends and application areas of fuzzy and hybrid methods to the risk assessment of construction projects. Advanced Engineering Informatics, 33, $112-131$

Kim, B.-C. (2015). Integrating risk assessment and actual performance for probabilistic project cost forecasting: a second moment Bayesian model. IEEE Transactions on Engineering Management, 62(2), 158-170.

Kokangül, A., Polat, U., \& Dağsuyu, C. (2017). A new approximation for risk assessment using the AHP and Fine Kinney methodologies. Safety Science, 91, 24-32.

Kuo, Y.-C., \& Lu, S.-T. (2013). Using fuzzy multiple criteria decision making approach to enhance risk assessment for metropolitan construction projects. International Journal of Project Management, 31(4), 602-614.

Leu, S.-S., \& Chang, C.-M. (2013). Bayesian-network-based safety risk assessment for steel construction projects. Accident Analysis \& Prevention, 54, 122-133.

Li, Z., Wen, G., \& Xie, N. (2015). An approach to fuzzy soft sets in decision making based on grey relational analysis and Dempster-Shafer theory of evidence: An application in medical diagnosis. Artificial Intelligence in Medicine, 64(3), 161-171.

Liu, J., Jin, F., Xie, Q., \& Skitmore, M. (2017). Improving risk assessment in financial feasibility of international engineering projects: A risk driver perspective. International Journal of Project Management, 35(2), 204-211.

Marcelino-Sádaba, S., Pérez-Ezcurdia, A., Lazcano, A. M. E., \& Villanueva, P. (2014). Project risk management methodology for small firms. International Journal of Project Management, 32(2), 327-340.

Muriana, C., \& Vizzini, G. (2017). Project risk management: A deterministic quantitative technique for assessment and mitigation. International Journal of Project Management, 35(3), 320-340.

Nasrabadi, M., \& Mirzazadeh, A. (2016). The inventory system management under uncertain conditions and time value of money. International Journal of Supply and Operations Management, 3(1), 1192-1214.

Niazi, M., Mahmood, S., Alshayeb, M., Riaz, M. R., Faisal, K., Cerpa, N., . . Richardson, I. (2016) Challenges of project management in global software development: A client-vendor analysis. Information and Software Technology, 80, 119.

Pan, Y., Zhang, L., Li, Z., \& Ding, L. (2019). Improved fuzzy Bayesian network-based risk analysis with interval-valued fuzzy sets and D-S evidence theory. IEEE Transactions on Fuzzy Systems, 28(9), 2063-2077.

Qazi, A., Quigley, J., Dickson, A., \& Kirytopoulos, K. (2016). Project Complexity and Risk Management (ProCRiM): Towards modelling project complexity driven risk paths in construction projects. International Journal of Project Management, 34(7), 1183-1198.

Sangaiah, A. K., Samuel, O. W., Li, X., Abdel-Basset, M., \& Wang, H. (2018). Towards an efficient risk assessment in software projects-Fuzzy reinforcement paradigm. Computers \& Electrical Engineering, 71, 833-846.

Suresh, K., \& Dillibabu, R. (2020). A novel fuzzy mechanism for risk assessment in software projects. Soft Computing, 24(3), 1683-1705.

Tang, H. (2015). A novel fuzzy soft set approach in decision making based on grey relational analysis and Dempster-Shafer theory of evidence. Applied Soft Computing, 31, 317-325.

Tao, S., Wu, C., Sheng, Z., \& Wang, X. (2018). Space-time repetitive project scheduling considering location and congestion. Journal of Computing in Civil Engineering, 32(3), 04018017.

Tonmoy, F. N., Wainwright, D., Verdon-Kidd, D. C., \& Rissik, D. (2018). An investigation of coastal climate change risk assessment practice in Australia. Environmental Science \& Policy, 80, 9-20.

Valipour, A., Yahaya, N., Md Noor, N., Antuchevičienė, J., \& Tamošaitienė, J. (2017). Hybrid SWARA-COPRAS method for risk assessment in deep foundation excavation project: An Iranian case study. Journal of Civil Engineering and Management, 23(4), 524-532.

Wang, C. M., Xu, B. B., Zhang, S. J., \& Chen, Y. Q. (2016). Influence of personality and risk propensity on risk perception of Chinese construction project managers. International Journal of Project Management, 34(7), 1294-1304.

Wang, J., Hu, Y., Xiao, F., Deng, X., \& Deng, Y. (2016). A novel method to use fuzzy soft sets in decision making based on ambiguity measure and Dempster-Shafer theory of evidence: an application in medical diagnosis. Artificial Intelligence in Medicine, 69, 1-11.

Williams, T. (2017). The nature of risk in complex projects. Project Management Journal, 48(4), 55-66.

$\mathrm{Wu}$, D. D., Chen, S.-H., \& Olson, D. L. (2014). Business intelligence in risk management: Some recent progresses. Information Sciences, 256, 1-7.

Yang, Y., Wang, J., Wang, G., \& Chen, Y.-W. (2019). Research and development project risk assessment using a belief rule-based system with random subspaces. Knowledge-Based Systems, 178, 51-60.

Yet, B., Constantinou, A., Fenton, N., Neil, M., Luedeling, E., \& Shepherd, K. (2016) A Bayesian network framework for project cost, benefit and risk analysis with an agricultural development case study. Expert Systems with Applications, $60,141-155$.

Zadeh, L. A. (1986). A simple view of the Dempster-Shafer theory of evidence and its implication for the rule of combination. A.I. Magazine, 7(2), 85-85.

Zavadskas, E. K., Turskis, Z., \& Tamošaitiene, J. (2010). Risk assessment of construction projects. Journal of Civil Engineering and Management, 16(1), 33-46. 
Zhang, Y. (2016). Selecting risk response strategies considering project risk interdependence. International Journal of Project Management, 34(5), 819-830.

Zhang, Y., \& Fan, Z.-P. (2014). An optimization method for selecting project risk response strategies. International Journal of Project Management, 32(3), 412-422.

Zou, Y., Kiviniemi, A., \& Jones, S. W. (2017). A review of risk management through BIM and BIM-related technologies. Safety Science, 97, 88-98.

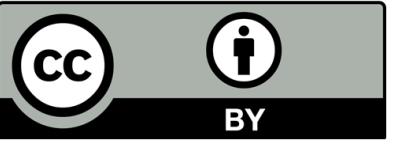

(C) 2022 by the authors; licensee Growing Science, Canada. This is an open access article distributed under the terms and conditions of the Creative Commons Attribution (CC-BY) license (http://creativecommons.org/licenses/by/4.0/). 